\title{
La inteligencia emocional en alumnos de educación escolar básica de la ciudad de Pilar, en el desarrollo de sus habilidades en contexto educativo
}

\author{
Vicente Ferrer González Rodríguez \\ gvicenteferrer@gmail.com \\ Universidad Nacional de Pilar \\ Pilar - Paraguay
}

\section{RESUMEN}

En la ciudad de Pilar, Departamento de Neembucú, se lleva adelante el presente trabajo de investigación, cuyo objetivo radica en conocer la inteligencia emocional en los alumnos de educación escolar básica de la ciudad de Pilar, en el desarrollo de sus habilidades en el ámbito educativo. Se toma como objeto de estudios los alumnos y docentes de las escuelas urbanas públicas subvencionadas de la ciudad vinculadas a la Fundación Redentorista de Obras Sociales y Educacionales de Pilar (FROSEP). Para alcanzar los objetivos y atendiendo la relevancia del tema, se busca dimensionar la importancia que alcanza la inteligencia intrapersonal e interpersonal y su influencia en el rendimiento académico de los alumnos de las mencionadas instituciones educativas. Para el efecto, se recurre a métodos y técnicas que corresponden a la naturaleza del estudio, ajustándose a los parámetros de un enfoque de investigación mixta, con un diseño de triangulación concurrente (DITRIAC). En este aspecto, el trabajo responde a un estudio del tipo descriptivo, recurriendo a técnicas de los métodos cuantitativo y cualitativo, lo que asegura una manera más confiable de recolección de datos. se destaca como hallazgo importante que existe correspondencia en aquellos alumnos que alcanzan niveles altos de inteligencia emocional y calificaciones altas. La misma situación acontece con respecto aquellos alumnos que presentan niveles medios de inteligencia emocional y calificaciones medias. Además, las percepciones personales que presentan los niños sobre sí mismos, así como la autoestima, son elevados, con habilidades sociales efectivas y de calidad

Palabras clave: inteligencia emocional; inteligencia interpersonal; inteligencia intrapersonal; rendimiento académico. 


\title{
Emotional intelligence in basic school education students in the city of Pilar, in the development of their skills in the educational context
}

\begin{abstract}
In the city of Pilar, Department of Neembucú, the present research work is carried out, whose objective is to know the emotional intelligence in students of basic school education in the city of Pilar, in the development of their skills in the educational field . The students and teachers of the subsidized public urban schools of the city linked to the Redemptorist Foundation of Social and Educational Works of Pilar (FROSEP) are taken as the object of study. To achieve the objectives and taking into account the relevance of the topic, it seeks to measure the importance of intrapersonal and interpersonal intelligence and its influence on the academic performance of students from the aforementioned educational institutions. For this purpose, methods and techniques that correspond to the nature of the study are used, adjusting to the parameters of a mixed research approach, with a concurrent triangulation design (DITRIAC). In this regard, the work responds to a descriptive study, resorting to quantitative and qualitative method techniques, which ensures a more reliable way of data collection. It stands out as an important finding that there is correspondence in those students who achieve high levels of emotional intelligence and high grades. The same situation occurs with respect to those students who present average levels of emotional intelligence and average grades. In addition, the personal perceptions that children present about themselves, as well as their self-esteem, are high, with effective and quality social skills
\end{abstract}

Keywords: emotional intelligence; interpersonal intelligence; intrapersonal intelligence; academic performance

Artículo recibido: 15 noviembre. 2021 Aceptado para publicación: 10 diciembre 2021 Correspondencia: gvicenteferrer@gmail.com Conflictos de Interés: Ninguna que declarar 


\section{INTRODUCCIÓN}

La inteligencia es considerada por muchos autores como una habilidad del ser humano que influye en su desempeño, en todas las tareas de referencia a la cualidad cognoscitiva. Dicho de esta manera, una persona inteligente hace un trabajo al resolver problemas, explicarlos o proponer encrucijadas.

Según Salovey y Mayer (1990), la inteligencia emocional es la capacidad para supervisar los sentimientos y las emociones de uno mismo y de los demás, discriminarla entre ellos y usar esta información para la orientación y el pensamiento propio (Bisquerra, Pérez y García, 2015, p. 53).

La inteligencia emocional como concepto ha sido utilizada a comienzos de la década de los 90. Desde entonces, ha irrumpido con una fuerza inusitada a todos los campos del conocimiento, siendo el ámbito educativo el espacio donde se la ha estudiado con mayor relevancia.

Es así que, en 1993 Howard Gardner, en su muy referenciada publicación hace alusión a siete estadios de inteligencia, vinculando directamente a la inteligencia interpersonal e intrapersonal con la inteligencia emocional. Dos años más tarde, Daniel Goleman llega a resaltar dicho término a través del éxito que alcanzó su libro.

Según Goleman (2012) la inteligencia emocional es una capacidad aprendida y susceptible de desarrollo mediante el entrenamiento en la percepción de uno mismo, autorregulación, automotivación, empatía y capacidad de relación (Rebollo Goñi y Peña Álvarez, 2017, p. 30).

Gardner (1993) propuso que la vida humana requiere del desarrollo de varios tipos de inteligencia: musical, lingüística, matemática, cinestésica, visual-espacial, naturalista, interpersonal y la inteligencia intrapersonal; estas dos últimas inteligencias se convierten en un anticipo de lo que sería más adelante la inteligencia emocional (Muñóz Prieto, 2017, p. 40).

La inteligencia interpersonal es la capacidad de entender a los demás e interactuar eficazmente con ellos, en tanto que la inteligencia intrapersonal es la capacidad de construir una percepción precisa respecto de sí mismo y de organizar y dirigir su propia vida (Alvarado y Salinas, 2018, p. 43).

Entonces, mientras la inteligencia interpersonal incluye la sensibilidad a expresiones faciales, la voz, los gestos y posturas y la habilidad para responder, la inteligencia 
intrapersonal incluye la autodisciplina, auto comprensión y autoestima.

La inteligencia interpersonal consiste en la capacidad de entender las intenciones, motivaciones y deseos de otras personas, la inteligencia intrapersonal consiste en la capacidad de entenderse a uno mismo, y apreciar las motivaciones, los sentimientos y los miedos que se sienten (Heredero y Garrido, 2017, p. 179).

Así mismo, la inteligencia interpersonal es visible en niños que disfrutan trabajando en grupo, que son convincentes en sus negociaciones con pares y mayores, que entienden al compañero. La inteligencia intrapersonal se evidencia en niños que son reflexivos, de razonamiento acertado y suelen ser consejeros de sus pares.

A partir de la popularización del concepto de inteligencia emocional surgieron diferentes modelos conformados en función de la visión particular de sus autores, éstos pueden clasificarse en dos grandes grupos: los modelos mixtos y el modelo de habilidad (Márquez Ávila, 2018, p. 6).

A grandes rasgos, los modelos mixtos, en su estudio aglutinan diversas habilidades, destrezas, objetivos, rasgos de personalidad, competencias y disposiciones afectivas. El modelo de habilidad que implica capacidades tales como las de supervisar los sentimientos y las emociones propias, así como las de los demás, de discriminar entre ellas, y usar la información para guiar el pensamiento y las acciones

Los principales exponentes de los modelos mixtos comprenden, en primer término la propuesta de Goleman, quién plantea la inteligencia emocional como un conjunto de características claves para resolver con éxito los problema, y la propuesta de Baron, quién supone que la inteligencia socioemocional es un conjunto de competencias y habilidades que establecen cuan acertadamente los individuos se entienden, comprenden a otros, expresan sus emociones y afrontan las demandas de la vida cotidiana.

El modelo de habilidad, sustentado por Salovey y Mayer, para quiénes la característica de la inteligencia social incluye un conjunto de capacidades que explican las diferencias individuales en el modo de percibir y comprender las emociones.

Hoy día, la Inteligencia Emocional constituye una variable de importancia crucial para la comprensión del éxito social de las personas, ante los múltiples desafíos que se presenta en el mundo. En este sentido, en la actualidad se hace necesario prestar mayor atención a las habilidades emocionales, al estudio de la conciencia y la vida afectiva. 
Desde esta perspectiva, la relevancia del estudio de la inteligencia emocional surge como un intento de considerar la importancia superlativa del papel que desempeñan las emociones en el intelecto humano, en la adaptación social del individuo, en su equilibrio personal y colectivo.

Entonces, el desarrollo de la inteligencia emocional en las personas genera una suerte de empatía, encaminado a facilitar y profundizar el enlace emergente entre los miembros del vínculo social próximo e inmediato; situación que pone de manifiesto lo fundamental que resulta para una vida social próspera.

Es así que, en la actualidad se considera a la inteligencia emocional como un factor relevante en la responsabilidad que juega para el éxito o el fracaso del individuo, sea cual fuere su ámbito de competencia, ya que lo encamina a manejar mejor las situaciones que enfrenta en la cotidianeidad de sus actividades.

Se puede decir que la inteligencia emocional se constituye en el uso inteligente de las emociones; es así que, de forma intencional, el individuo hace que sus emociones trabajen con el propósito de ayudar a guiar su comportamiento en la búsqueda de la mejora de sus resultados.

Payne en 1996 sería el primero en utilizar el término Inteligencia emocional en el ámbito estrictamente educativo al proponer integrar emoción e inteligencia para enseñar en las escuelas respuestas emocionales a los niños y evitar así conductas destructivas (Egocheaga León, 2017, p. 22).

Desde entonces, el concepto ha sido intensamente estudiado, siendo reformulado y adecuado a los alcances sociales y académicos. Desde este punto de vista, la utilidad de la inteligencia emocional no es nuevo en el ámbito educativo, siendo ampliamente estudiado sus alcances, limitaciones e influencias.

Es precisamente, en la etapa escolar donde la inteligencia emocional adquiere relevancia, ya que un estudiante que no llega a disponer de estrategias y competencias específicas, encuentran severas dificultades en la tarea de sortear con éxito las exigencias y demandas emergentes en el ámbito.

Un fracaso en este aspecto, relativo a una experiencia adversa, podría desencadenar en el estudiante actitudes negativas, lo que podría derivar en la falta de interés en proseguir sus estudios. 
Las personas emocionalmente inteligentes son aquellas capaces de atender a las emociones percibidas en su contexto más próximo, comprendiendo sus posibles causas y consecuencias y desarrollando, así, las estrategias necesarias para regular o manejar los diferentes estados emocionales (Usán y Salavera, 2018, p. 97).

En el contexto del ámbito educativo, los alumnos deben ser capaces de desarrollar dichas estrategias necesarias para enfrentar con éxito los desafíos e implicancias que conllevan las actividades áulicas.

De ahí que, la formación de las capacidades emocionales y sociales en los escolares puede conducir a una reducción significativa de las conductas agresivas que llevan a la violencia, en las instituciones educativas.

Las habilidades que intervienen en la inteligencia emocional comprenden: conocer nuestros propios sentimientos, experimentar empatía, aprender a manejar las emociones, reparar el daño emocional, y combinarlo todo (Porcayo Dominguez, 2013, p. 27).

El modelo basado en la habilidad inteligencia emocional incluye cuatro grandes componentes: la percepción, evaluación y expresión de la emoción, el uso de las emociones para facilitar el pensamiento, el entendimiento y análisis de la información emocional, y regulación de la emoción (Vargas Paredes, 2018, p. 32).

La inteligencia emocional se convierte en una posibilidad para acercarse a comprender los procesos de convivencia que ocurren en el contexto escolar y explorar formas de relación diferentes que favorezcan la interacción cotidiana dentro de este (Camacho Bonilla y otros, 2017, p. 26).

La desatención de los docentes en la aplicación de estrategias didácticas específicas conduce necesariamente a inadecuadas resoluciones de conflictos emocionales de los alumnos, que normalmente se constituyen en factores causantes de malestares fisiológicos y sociales.

El principio que rige el desarrollo de la inteligencia emocional en las relaciones de los estudiantes, encaminados por los docentes, es el respeto por las emociones y sentimientos emergentes en el aula, lo que supone el progreso de un ambiente de empatía, propiciado en la capacidad de transmitir sensaciones.

Este ambiente propuesto por el docente favorecido por el uso de las herramientas para el logro del desarrollo de la inteligencia emocional, posibilita alcanzar progresos 
importantes en el proceso de enseñanza aprendizaje, lo que conduce a los escolares favorablemente al perfeccionamiento de sus habilidades.

Un maestro emocionalmente inteligente debe percibir este movimiento afectivo para dirigirlo de forma provechosa al aprendizaje, basándose en su capacidad interpersonal y liderazgo. Un experto motivador, conciliador y con buen sentido del humor tendrá un impacto positivo en sus alumnos (Bravo Sánchez, 2018, p. 467).

Las exigencias de los nuevos modelos educativos emergentes demandan docentes emocionalmente inteligentes para ejercer su profesión, comprometidos en el aspecto afectivo y personal, capaces de entender la conducta emocional de sus alumnos y conducirlos conscientemente en el desarrollo social afectivo.

El docente debe presentarse como un profesional preparado para enfrentar las demandas emergentes en el contexto escolar, donde las emociones ejercen un valor preponderante en la formación de los educandos.

La capacidad de los educadores para saber estimular la disposición emocional del alumno determinará en gran medida, su aprendizaje y logro académico (Extremara, Rey y Pena, 2018, p. 72).

El profesor no sólo tiene la obligación de conocer las materias que explica y los métodos de enseñanza-aprendizaje, sino que debe ocuparse también de comprender a los estudiantes, de interesarse por su espacio vital, por sus emociones, por lo que sienten, por lo que piensan (Fernández Domínguez, Palomero Pescador y Teruel Melero, 2009, p. 34). Desde esta perspectiva, la inteligencia emocional se constituye es un aspecto primordial para el docente, teniendo en cuenta que se debe fundar en el proceso de su formación inicial y permanente. En este aspecto, resalta fehacientemente que la eficiencia de la labor docente tiene mucho que ver con la capacidad desarrollada en comprender los estados emocionales de sus estudiantes.

Las emociones juegan un papel importante en la adaptación de los estudiantes en cuanto al manejo emocional de aquellas variables contextuales y personales que se producen y que determinan cuestiones relevantes como el bienestar personal, la felicidad académica, la interacción grupal o el rendimiento escolar, entre otros (Usán, y Salvera, 2019, p. 9). El interés relativo respecto al estudio de tema se centra en dimensionar la importancia que tiene la inteligencia emocional en el ámbito educativo y como ella trasciende para la vida futura. Teniendo en cuenta los objetivos de la investigación que se pretende llevar 
adelante en el presente trabajo, su resultado permitirá de dimensionar la importancia de la inteligencia emocional en el ámbito educativo.

Precisamente, el presente trabajo tiene por propósito conocer la inteligencia emocional en los alumnos de educación escolar básica de la ciudad de Pilar, Departamento de Ñeembucú, en el desarrollo de sus habilidades en contexto educativo. Se considera, para el efecto, las escuelas urbanas públicas subvencionadas de la ciudad vinculadas a la Fundación Redentorista de Obras Sociales y Educacionales de Pilar (FROSEP).

Más específicamente, se busca determinar los niveles de inteligencia intrapersonal e interpersonal y su influencia en el rendimiento académico de los alumnos de las mencionadas instituciones educativas.

Para hacer factible el presente trabajo, se recurre a métodos y técnicas que corresponden a la naturaleza del estudio, ajustándose a los parámetros de un enfoque de investigación mixta, con un diseño de triangulación concurrente (DITRIAC).

Así mismo, el trabajo responde a un estudio del tipo descriptivo, recurriendo a técnicas de los métodos cuantitativo y cualitativo, lo que asegura una manera más confiable de recolección de datos.

\section{ESTRATEGIAS METODOLÓGICAS O MATERIALES Y MÉTODOS}

Para el logro de los objetivos propuestos, La investigación se ubica dentro del enfoque mixto, con un diseño de triangulación concurrente (DITRIAC).

Se utiliza cuando el investigador pretende confirmar o corroborar resultados y efectuar validación cruzada entre datos cuantitativos y cualitativos, así como aprovechar las ventajas de cada método y minimizar sus debilidades (Hernández Sampieri y otros, 2014, p. 557).

El tipo de investigación en la que un investigador o equipo de investigadores combina elementos de enfoques de investigación cualitativa y cuantitativa (por ejemplo, uso de puntos de vista cualitativos y cuantitativos, recopilación de datos, análisis, técnicas de inferencia) (Gallardo Echenique, 2017, p. 26).

La investigación mixta pretende conjugar los procedimientos de la investigación cuantitativa con lo de la investigación cualitativa, en el convencimiento de que reduccionismo, el extremismo en la investigación no conducen a nada bueno (Ñaupas Paitán y otros, 2018, p. 142). 
El objetivo de este tipo de diseño es combinar las fortalezas de ambas metodologías para obtener datos acerca de un mismo problema de investigación y que puedan complementarse. El estudio se realiza en una sola etapa en la que simultáneamente se recolecta, procesa y analiza la información obtenida.

Con respecto al estudio cualitativo, se pone de manifiesto su utilidad por cuanto ofrece técnicas especializadas para obtener respuestas a fondo, acerca de lo que las personas piensan y sienten, presentando como resultado informaciones válidas que orientan la comprensión más allá de la descripción con palabras.

A través de las técnicas de este método se emprende sondear entrevistados para describir con propiedad las diferentes situaciones presentadas en cuanto a la Inteligencia Emocional y su importancia en el contexto educativo.

En lo que se refiere a los datos cuantitativos, que produce datos numéricos, se pretende exponer datos numéricos estadísticos a los efectos del requerimiento del presente trabajo. El trabajo se encamina a establecer la influencia de la inteligencia emocional en el ámbito educativo, específicamente en lo que refiere al rendimiento académico, utilizando como parámetros las matemáticas y lengua materna castellana, enmarcadas en el contexto de las llamadas teorías del factor $\mathrm{G}$ de la inteligencia tradicionalmente conocida.

La presente investigación se orienta principalmente en aplicar las herramientas de recolección de datos a los alumnos y docentes del segundo ciclo de la Educación Escolar Básica, turnos mañana y tarde, de las escuelas San Blas, San Gerardo y San José ciudad de Pilar.

El estudio se desarrolla en las mencionadas Escuelas del nivel primario de la zona urbana de la ciudad de Pilar, que forman parte de la administración de la Fundación Redentorista de Obras Sociales y Educacionales de Pilar (FROSEP).

Estas Instituciones Educativas Públicas son de Gestión Privada Subvencionadas que cuentan con 3.345 alumnos y 390 profesionales de la Educación, que incluyen a directores, Docentes, Técnicos y Funcionarios Administrativos y de Servicios con rubros oficiales y privados, que prestan servicios educativos en los diferentes niveles del Sistema Educativo Nacional.

Lo citado en el párrafo anterior es considerado como población a los efectos de la presente investigación. Para obtener la muestra se recurre al modelo propuesto por Bernal (2010, p. 52). 
Para el efecto, según la fórmula propuesta correspondiente a la población finita, se concluye sobre el tamaño mínimo de muestra representativa, especificando los valores.

De esta manera, los datos de la población se someten a la siguiente fórmula:

$$
n=\mathbf{Z}^{2}{ }^{a} / 2 * N^{*} p^{*} q / \mathbf{E}^{2}(\mathrm{~N}-1)+\mathbf{Z}^{2}{ }^{2} / 2 * \mathbf{p}^{*} q
$$

\section{Donde}

$n=$ tamaño necesario de la muestra

$\mathrm{Z}=$ Margen de confiabilidad

$\mathrm{S}=$ desviación estándar estimada

$\mathrm{E}=$ Error o diferencia máxima

$q=$ error de estimación

Obteniéndose como resultados una muestra representativa de 247 alumnos y 107 docentes, con un nivel de confianza del $95 \%$ y 6,0\% como estimación del error.

El tipo de muestreo utilizado corresponde al probabilístico, más específicamente al alzar simple y sistemática, que tiene como fundamento en el principio de equiprobabilidad, en el cual todos los individuos de la muestra seleccionada, tienen las mismas probabilidades de ser elegidos.

Con respecto a las técnicas de recolección de datos, se utilizó un formulario impreso, destinados a obtener respuestas sobre el problema en estudio y que el investigado lleva por sí mismo. En este caso se utilizó preguntas estructuradas, que fueron aplicados a los alumnos.

El instrumento de evaluación de elaboración propia, estuvo basado en las cinco competencias emocionales propuestas por el Doctor Daniel Goleman, de las cuales se desprenden las preguntas que determinan, tanto la inteligencia intrapersonal como la interpersonal, y adecuado según el lenguaje infantil y considerando la mayor fidelidad posible a las posibles situaciones emocionales que los niños pudieran experimentar.

Estas preguntas incluidas en el cuestionario fueron elaboradas a partir de los objetivos definidos y en el que fueron considerados los términos adecuados que permitan la comprensión del evaluado al momento de marcar su respuesta.

Para la establecer la escala a utilizar se procedió a la distribución proporcional de los ítems procediendo a la suma de la valoración máxima que pudiese obtenerse tanto para determinar el nivel de inteligencia emocional que incluye los veinticinco ítems entre tres valoraciones que son; la inteligencia emocional alta, media y baja. 
El mismo proceso establecido para las divisiones de la inteligencia emocional las cuales son la inteligencia intrapersonal y la inteligencia interpersonal. Para todos los ítems, las valoraciones de los planteamientos presentan opciones del uno al seis según desde el desacuerdo absoluto hasta el total acuerdo con lo planteado. Así tenemos a los doce primeros ítems correspondientes a las competencias personales y del trece al veinte y cinco a las competencias sociales, cada uno de ellos con sus respectivos indicadores.

Para el procedimiento de validación y una vez concluido con la elaboración de los instrumentos de recolección de datos, se sometió a una prueba piloto a alumnos para lo cual se seleccionó bajo un criterio arbitrario un aproximado de 40 alumnos que representa un número mayor que el número de ítems.

Se procedió a la aplicación del cuestionario hasta completar el número necesario. De los 40 a se eliminaron aquellas en las que los sujetos no respondieron o eran evasivos, quedando finalmente la muestra reducida a un número de 34 (treinta y cuatro) escolares. Así mismo, se procedió a la revisión de documentos, que corresponden a los registros de acontecimientos ocurridos recientes o pasados. Estos documentos son fuentes originales de informaciones.

Se recurrió a documentos oficiales de las instituciones educativas investigadas tales como planillas de calificaciones finales. En el análisis de estas fuentes primarias fueron tomados con precaución para garantizar que los documentos sean auténticos.

\section{RESULTADOS Y DISCUSIÓN}

Los niveles de inteligencia emocional que fueron establecidos a través de un instrumento de elaboración propia basado en veinticinco competencias emocionales que demuestran la influencia que tiene la inteligencia emocional sobre el rendimiento académico.

Es así que, se pudo verificar que, a medida en que se registran niveles altos de inteligencia emocional en los alumnos seleccionados, estos presentan niveles altos de calificaciones finales. Esta misma situación se presenta, de manera proporcional para aquellos estudiantes con niveles medios de inteligencia emocional. Cabe mencionar que, no fueron identificados casos que puedan adquirir relevancia en relación a alumnos con niveles bajos de inteligencia emocional, basados siempre en las tres escalas establecidas en el cuestionario.

El logro de un nivel alto de inteligencia emocional implica también una realización personal elevada, que determina para los escolares el suficiente conocimiento de sí 
mismos, que les permite identificar sus limitaciones y sus aspectos personales más desarrollados, la manera de aprovecharlos como la forma en que lo aplican ante cada situación que se les presentan, en los contextos estrictamente educativos como así también en sus relaciones sociales.

Se acota, que ambas competencias se combinan al no poder establecerse en formas aisladas, funcionando de manera superpuestas o bien utilizadas permanentemente y en forma continua.

Se determinó el nivel de inteligencia emocional desde el punto de vista de sus aspectos generales y bien diferenciados que corresponden básicamente al manejo de las emociones desde el conocimiento propio para proyectarse a las relaciones sociales. Los alumnos demostraron suficientes habilidades emocionales al identificar los momentos claves y la manera en que pueden llegar a influir en sus decisiones finales.

Los niños con una inteligencia emocional alta, presentaron rasgos que tienden a un mejor aprendizaje, con menos o nulos problemas de conducta; las percepciones personales sobre sí mismos, así como la autoestima son elevados, con habilidades sociales efectivas y de calidad utilizadas cotidianamente en todos los ámbitos, principalmente el educativo y familiar. Estos alumnos son menos violentos, con tendencia a identificar sentimientos de sus contactos sociales y, en general, resuelven con asertividad sus conflictos presentados. Al determinar el nivel de inteligencia emocional según el sexo, no se llegó a verificar diferencias importantes significativas. En general, se comprueba un promedio alto entre varones y mujeres. Sin embargo, es de importancia destacar diferencias sutiles a partir de casos muy específicos que no fueron establecidos por carecer de parámetros vinculados al entorno familiar, que puedan determinar inconvenientes dentro del mismo, en sus diversas acepciones y que pudieran tener implicancia en sus emociones.

En la presente investigación, en particular se establece la influencia de la inteligencia emocional en el ámbito educativo a partir de dos fuentes utilizadas.

Al estudiar una muestra muy interesante en estas tres escuelas, se ha realizado como un cierto paralelismo entre la actitud de los escolares actuales y los de unas décadas atrás; como la chispa del propio ser único de cada niño sigue inherente en ellos, esto sin definir su origen familiar o económico, por ejemplo, está incorporado en los mismos el elemento de la información extensa que manejan. 
Es riesgoso aventurarse en decir que se vuelven adultos a temprana edad, sino a diferencia de generaciones anteriores observamos justamente a niños y pre adolescentes más abiertos en expresar sus emociones.

\section{CONCLUSIÓN O CONSIDERACIONES FINALES}

Como parte del alcance de los objetivos propuestos fueron determinados los niveles de inteligencia emocional de la muestra establecida en las tres escuelas investigadas. Así mismo, derivado del nivel general de inteligencia emocional fueron establecidos los niveles de inteligencia intrapersonal e inteligencia interpersonal el cual fue puesto en relación con los niveles de rendimiento académico final.

En este aspecto, se destaca como hallazgo importante que existe correspondencia en aquellos alumnos que alcanzan niveles altos de inteligencia emocional y calificaciones altas. La misma situación acontece con respecto aquellos alumnos que presentan niveles medios de inteligencia emocional y calificaciones medias.

Se comprueba, además, que las percepciones personales que presentan los niños sobre sí mismos, así como la autoestima, son elevados, con habilidades sociales efectivas y de calidad, utilizadas cotidianamente en todos los ámbitos, principalmente el educativo y familiar.

Por otra parte, se evidencia que los alumnos demostraron suficientes habilidades emocionales al identificar los momentos claves y la manera en que pueden llegar a influir en sus decisiones finales.

La teoría de la inteligencia emocional ofrece indicadores para el estudio de competencias específicas que permiten identificar en primer lugar los aspectos a potenciar y, posterior a ello, establecer técnicas de abordaje regidos necesariamente por la disponibilidad del equipo psicopedagógico en las escuelas; en ausencia de éste se requiere un buen tacto del docente de aula para identificar aquellas competencias emocionales que requieren elevar su desarrollo, al tiempo de llevar adelante una atención personalizada.

Todo ello, enfocado a fortalecer las valoraciones de sí mismos, derivado del conocimiento de las propias emociones, proyectado hacia una efectiva práctica de las relaciones sociales.

El aprendizaje resulta efectivo en situaciones de conformidad, en referencia al ambiente en el aula, que debe ser lo más motivable posible, en contraposición a conceptos 
anteriores que la misma tendría que partir de un factor externo, que en este caso es el docente.

No obstante, la teoría de la inteligencia emocional, entre sus distintas dimensiones menciona la capacidad que tiene el niño de generar la suficiente automotivación como para regular internamente e independientemente ciertos factores externos.

La evaluación y valoración de los resultados de los alumnos por parte de los profesores resulta fundamental para que los niños se sientan capaces de creer que ellos pueden académicamente ir construyendo en cada clase una formación con bases sólidas, pues una interpretación negativa de las intenciones o capacidades de los alumnos tendrían como desencadénate una subvaloración de sí mismos.

\section{LISTA DE REFERENCIAS}

Alvarado, L.H. y Salinas, P.V. (2018). Programa lúdico "PALEIN" para desarrollar la inteligencia intrapersonal e interpersonal de las niñas de $1^{\circ}$ grado de educación primaria de la I.E N 81007 “Modelo"-Trujillo, 2017. Tesis. Universidad Nacional de Trujillo. Facultad de Educación y Ciencias de la Comunicación. Trujillo, Perú.

Bernal Tórrez, C. (2010). Metodología de la investigación. Pearson educación, Tercera edición. Colombia.

Bravo Sánchez, Y.B. (2018). Inteligencia emocional como base fundamental de la gestión pedagógica. Dominio de las Ciencias 2477-8818 Vol. 4, núm. 1. Enero, 2017, pp. 465-472. https://dx.doi.org/10.23857/dom.cien.pocaip.2017.4.1.enero.465-472

Bisquerra, R., Pérez-González, J. C., y García Navarro, E. (2015). Inteligencia emocional en educación. Madrid: Síntesis.

Camacho Bonilla y otros (2017). Convivencia escolar y cotidianidad: una mirada desde la inteligencia emocional. Revista Educación y Desarrollo Social, 11(1), 24-47. DOI: org/10/18359/reds.2649.

Egocheaga León, C.R. (2017). Inteligencia emocional y la convivencia escolar en estudiantes del tercer grado de secundaria de la Institución Educativa Ciro Alegría, Carabayllo-2017. Tesis. Universidad César Vallejo. Escuela de Post Grado. Lima, Perú. 
Extremara, N. Rey, y Pena, M. (2018). Educadores de corazón. Inteligencia emocional como elemento clave en la labor docente. adres Y Maestros / Journal of Parents and Teachers, (368), 65-72. https://doi.org/10.14422/pym.i368.y2016.011

Fernández Domínguez, M.R., Palomero Pescador, J.E. y Teruel Melero, M.P. (2009). El desarrollo socioafectivo en la formación inicial de los maestros. REIFOP, 12 (1), 33-50. Enlace web: http://www.aufop.com - Consultada en fecha (dd-mm-aa):

Gallardo Echenique, E. E. (2017). Metodología de la Investigación: manual autoformativo interactivo. Universidad Continental, 1ra Edic. Huancayo, Perú.

Heredero, E.S. y Garrido, M.P. (2017). Desarrollo de la inteligencia interpersonal e intrapersonal en educación primaria a partir del uso de tecnologías de información y comunicación: estudio de casos. Notandum 44-45 mai-dez 2017 - CEMOrOCFeusp / IJI-Univ. do Porto. DOI: http://dx.doi.org/10.4025/notandum.44.15

Hernández Sampieri, R., Fenandez Collado, C. y Baptista, M. (2014). Metodología de la investigación. McGrawHill education, Sexta edición. México.

Muñóz Prieto, M.M. (2017). Desarrollar la inteligencia emocional para prevenir el acoso escolar. EA, Escuela Abierta, 20, 35-46. doi:10.29257/EA20.2017.04. En https://ea.ceuandalucia.es/index.php/EA/article/view/12

Ñaupas Paitán, H. y otros. (2018). Metodología de la investigación cuantitativacualitativa y redacción de la tesis. 5a. Edición. Bogotá: Ediciones de la U, 2018, p. $562 ; 24 \mathrm{~cm}$.

Porcayo Dominguez, B. (2013). Inteligencia emocional en niños. Tesis. Universidad Autónoma del Estado de México Facultad De Ciencias De La Conducta. Toluca, México.

Rebollo Goñi, E. y Peña Álvarez, C. (2017). Estudio de la Inteligencia Emocional y Función Ejecutiva en Educación Primaria. ReiDoCrea, 6, 29-36. En https://dialnet.unirioja.es/servlet/articulo?codigo $=5908618$

Usán, P. y Salvera, C. (2019). El rendimiento escolar, la inteligencia emocional y el engagement académico en adolescentes. Universidad de Almería. Electronic Journal of Research in Educational Psychology, 17 (1), 5-26. ISSN:1696-2095. 2019. no. 47. En http://repositorio.ual.es/bitstream/handle/10835/7547/18797048-1-PB.pdf?sequence $=1$ 
Usán, P. y Salvera, C. (2018). Motivación escolar, inteligencia emocional y rendimiento académico en estudiantes de educación secundaria obligatoria. Actualidades en Psicología, $\quad 32(125), \quad$ 2018, 95-112. En http://revistas.ucr.ac.cr/index.php/actualidades

Vargas Paredes, 2018). Autoestima e inteligencia emocional en alumnos del sexto grado de la I.E. N 82023 “Otuzco Alto”, Centro Poblado Otuzco, Cajamarca en el año 2017. Tesis. Universidad Privada Antonio Guillermo Urrelo. Facultad de Piscología. Cajamarca, Perú. 